Archives de sciences sociales des religions

135 | juillet - septembre 2006

Réveils du soufisme en Afrique et en Asie

\title{
La Ahmadiyya au Bénin
}

Denise Brégand

\section{OpenEdition}

Journals

Édition électronique

URL : http://journals.openedition.org/assr/3737

DOI : 10.4000/assr.3737

ISSN : $1777-5825$

Éditeur

Éditions de l'EHESS

Édition imprimée

Date de publication : 1 septembre 2006

Pagination : 73-90

ISBN : 2-7132-2093-9

ISSN : 0335-5985

Référence électronique

Denise Brégand, «La Ahmadiyya au Bénin », Archives de sciences sociales des religions [En ligne], 135 | juillet - septembre 2006, mis en ligne le 16 octobre 2009, consulté le 19 avril 2019. URL : http:// journals.openedition.org/assr/3737 ; DOI : 10.4000/assr.3737 


\section{Denise Brégand}

\section{La Ahmadiyya au Bénin ${ }^{1}$}

La Ahmadiyya, premier mouvement musulman prosélyte et transnational a pu, à la faveur du renouveau démocratique des années 1990 au Bénin, y installer des missions et développer ses actions prosélytes et humanitaires. Cet article présente les stratégies de la Abmadiyya dans deux contextes différents, d'une part celui des villes côtières, Cotonou et Porto-Novo, où l'islam est minoritaire, d'autre part celui des départements du Nord du pays, où il est religion dominante en expansion ${ }^{2}$. Les premières enquêtes ont été effectuées en vue d'une communication au séminaire "Minorités en miroir : Musulmans en contexte non musulman, non-musulmans en contexte musulman » de l'IISMM en mai 2002, et actualisées au cours d'une mission en janvier et février $2005^{3}$.

$\mathrm{Au}$ Bénin où règne une bonne cohabitation entre les religions, parmi une floraison de courants chrétiens et dans une moindre mesure de courants musulmans, la Abmadiyya fait figure d'exception, car là comme partout dans le monde, les autres musulmans ne reconnaissent pas ses membres comme faisant partie de l'Umma. Il existe certes d'autres courants minoritaires, mais tous les musulmans se retrouvent sur la même place ' $\hat{\imath} d \hat{\imath}^{4}$ le jour des grandes fêtes musulmanes, alors que les Ahmadis ne partagent avec eux aucun lieu ni aucun moment. De ce fait, ce courant qui entend réformer l'islam trouve paradoxalement ses interlocuteurs chez les non musulmans.

Une première partie, tout en rappelant les spécificités du mouvement (doctrine, structure, prosélytisme) montrera leur application au Bénin, dans un contexte politique et religieux que présentera la deuxième partie. La troisième partie comparera les stratégies déployées en situation d'islam minoritaire dans les villes du Sud et en situation d'islam majoritaire dans le Nord du pays.

1. Mes remerciements à Marc Gaborieau pour sa lecture attentive et ses conseils.

2. À Porto-Novo : 25,1 \% de musulmans, à Cotonou : 14,2\%, à Parakou : 56,5\%, à Malanville : 94,5\%. Source : recensement de 2002.

3. Mes remerciements également à l'amîr (responsable national en poste à Porto-Novo) Assan Secundar et à son successeur Khalid Maamoud, qui m'ont toujours très bien reçue, manifestant à mon égard la plus grande ouverture d'esprit, et qui ont facilité mes recherches.

4. Le terrain sur lequel se rassemblent tous les musulmans lors des grandes fêtes. 


\section{Un mouvement transnational et prosélyte}

La Ahmadiyya au Bénin se rattache à la branche de Qadian ${ }^{5}$ où est né (vers 1838-1908), Mirzâ Ghulâm Ahmad, fondateur du mouvement. En 1882, à l'aube du XIV siècle A.H., dans l'Inde coloniale, dans un contexte de détérioration de la foi et des pratiques religieuses et de prosélytisme des missionnaires chrétiens, il reçoit le message divin qui lui confie la tâche de restaurer l'islam dans sa pureté. Il se déclare mujaddid (rénovateur), muhaddath (à qui Dieu parle) et mahdî (guide). Selon Yohanan Friedmann, il trouve son inspiration dans la littérature mystique médiévale, particulièrement dans l'œuvre d'Ibn Arabi chez lequel il puise l'idée centrale de la Abmadiyya, celle de la prophétie ininterrompue : il fait partie d'une chaîne de personnalités inspirées par Dieu pour assurer la mise en œuvre de sa volonté sur terre ${ }^{6}$. Dans cette perspective, de même que Jésus est le dernier de la chaîne qui commence avec Moïse pour les juifs, Mirzâ Ghulâm Ahmad est le dernier de la chaîne qui commence avec Mahomet : ainsi s'établit la similitude entre lui et Jésus. C'est là le point crucial de désaccord avec les sunnites pour lesquels il ne saurait y avoir de prophète après Mahomet. Mirzâ Ghulâm Ahmad développe l'idée selon laquelle Jésus n'est pas mort sur la croix ; il s'est seulement évanoui et a été sauvé par Allah, puis est parti en Inde où il est mort et enterré à Srinagar. Un mujaddid est à chaque siècle envoyé sur terre pour régler les problèmes de la communauté : à la fin du XIX ${ }^{\mathrm{e}}$ siècle l'islam se trouve confronté au prosélytisme chrétien, ce qui apporte à Mirzâ Ghulâm Ahmad une justification pour se déclarer «messie promis ", titre associé à celui de Jésus, ce que confirme une révélation divine le proclamant "Jésus revenu ». Il se présente également comme un avatar de Krishna : Allah a révélé que Krishna était " un homme parfait» qui nettoya l'Inde du vice ${ }^{7}$, et selon une prédiction trouvée dans des livres hindous, un avatar ayant des caractéristiques similaires à celles de Krishna doit apparaître à la fin des temps, une révélation apprend à Mirzâ Ghulâm Ahmad qu'il est cette personne ; cette idée de «l'homme parfait » trouve également sa source dans la pensée soufie. Pour Friedmann, comme pour Spencer Lavan ${ }^{8}$, Mirzâ Ghulâm Ahmad propose cette réforme en réaction au contexte de l'époque qui voit l'islam confronté à un double défi, d'une part l'action des missionnaires chrétiens, d'autre part, la fondation du mouvement réformiste hindou Arya Samaj.

Face aux attaques de ces derniers qui accusent l'islam de violence, Mirzâ Ghulâm Ahmad appelle les musulmans à renoncer à la guerre sainte et à se

5. En 1914, une scission survint entre la branche principale, dite Qadiani, et ceux qui s'en séparèrent pour former la branche de Lahore. Pour les Lahoris, Mirzâ Ghulâm Ahmad n'est pas un prophète, mais seulement un mujaddid (rénovateur) et ils ne vénèrent pas le calife du mouvement.

6. Friedmann, 1989, p. 106.

7. Idem, p. 123.

8. Lavan, "Sources for Ahmadiyya history ", in Gustafson et Kenneth W. Jones, 1975, p. 88 . 
consacrer au prosélytisme pacifique. Il encourage l'action missionnaire dans tout l'empire britannique et au-delà, un programme qui sera mis en œuvre par ses successeurs. La Ahmadiyya fit preuve, entre les deux guerres, d'un prosélytisme actif et efficace, " on a pour la première fois une institution religieuse purement transnationale, indépendante de tout support étatique, capable de gagner des convertis parmi les Anglais comme chez les Noirs de Chicago ou du Nigeria. Non seulement elle est indépendante des États, mais elle s'est donné la doctrine théologique qui légitime cette indépendance. Le jihâd a laissé la place à la prédication $\left(d a^{6} w a\right) »{ }^{9}$.

C'est ainsi que la Abmadiyya arriva en Afrique orientale et en Afrique occidentale en 1915 à Lagos, Accra et en Sierra Leone ${ }^{10}$. À partir de ces premières installations dans le Golfe de Guinée, des missionnaires furent envoyés plus au Nord, et dans les pays voisins. Très ancrée dans l'Afrique de l'Ouest anglophone ${ }^{11}$, elle est présente en Sierra Leone, Liberia, Burkina Faso, Togo, Gambie, Bénin. Le mouvement a été chassé en août 2001 de Guinée Bissau où il était présent depuis 1995, au motif qu'il «créait de sérieux malentendus au sein de la communauté musulmane ${ }^{12}$. Au Bénin, la Ahmadiyya a été introduite à Porto-Novo depuis le Nigeria au début des années 1960.

\section{Un oxymoron au sein de lisslam : \\ des fondamentalistes non orthodoxes et modernistes}

Les membres de la Abmadiyya partagent les points communs aux Qadianis $\mathrm{du}$ monde entier, mais comme toujours, les acteurs réinterprètent des normes religieuses importées. Ainsi, ils vénèrent Mirzâ Ghulâm Ahmad comme prophète : la prononciation de son nom, comme celle de Mahomet, est toujours suivie dévotement de la formule "sallallahu 'alaibi wa sallam", formule qui rythme leurs discours. Dans la déclaration que remplit le nouvel adepte, s'engageant à obéir au calife installé à Londres, la profession de foi stipule : " Je considère Muhammad comme le sceau des Prophètes, et je crois également à tout ce qu'a affirmé le Prophète Ahmad de Qadian ». Cette reconnaissance de Mirzâ Ghulâm Ahmad comme prophète n'est pas seulement à l'origine de la scission au sein du mouvement, elle est aussi la cause principale du rejet de la part des autres musulmans. En 1974, un amendement à la constitution du Pakistan déclare nonmusulmans ceux qui ne croient pas que Mahomet est le dernier des prophètes. Cette même année, un arrêté du gouvernement saoudien exclut les Ahmadis de l'Umma et leur interdit le pèlerinage à la Mecque.

9. Gaborieau, 2001 , p. $35-48$; cit. p. 38.

10. Fisher, 1963 ; AJAYI et CROWDER, 1988.

11. Selon Smith W. Cantwell (1956) le mouvement comptait un demi-million d'adhérents dans le monde. Il est très difficile aujourd'hui d'obtenir des données fiables, la Ahmadiyya faisant des déclarations exagérées.

12. Site internet Africa Online. 
Les Ahmadis sont pacifistes et insistent dans leurs tracts, dans leurs discours, sur leurs sites internet, sur le sens de jihâd comme « effort sur soi-même en vue de perfectionnement moral et religieux » et condamnent la notion de guerre sainte que Mirzâ Ghulâm Ahmad a demandé d'abandonner. C'est certainement le thème de réflexion le plus développé depuis le 11 septembre. Dans son sermon prononcé sur la place 'îdî le jour de Tabaski 2002, l'amîr ${ }^{13}$ a explicitement condamné l'action suicide au nom de l'islam.

Peut-on qualifier les Ahmadis de fondamentalistes ? Ils le sont dans la mesure où ils prônent un islam épuré de tout ce qui n'est pas écrit dans le Coran et rejettent les interprétations des juristes. Mirzâ Ghulâm Ahmad est venu pour ramener les musulmans dans la bonne voie: "Il énonce l'essence de l'islam, débarrassé de toutes les incrustations dont le corps de l'islam a été recouvert au cours des siècles, qui l'ont dégradé et défiguré comme elles ont dénaturé la société musulmane ${ }^{14}$. Leur refus de tout ancrage dans les cultures - thème développé par tous les fondamentalistes - n'est pas sans poser de problèmes au Bénin. En effet, quand un enfant naît, les musulmans célèbrent la 'aqîqa sept jours après la naissance ; tout décès est suivi de cérémonies, huit jours, quarante jours, trois mois après. Ces cérémonies très onéreuses rythment la vie collective, vouloir les supprimer ne vise pas seulement une pratique religieuse, mais également les règles de sociabilité et de contrôle qu'exercent les groupes d'appartenance sur leurs membres ${ }^{15}$. La Abmadiyya dénonce aussi le fait que les imâms se fassent payer pour les baptêmes, mariages et cérémonies post-mortuaires, et que les alfa ${ }^{16}$ gagnent de l'argent avec les talismans. Elle partage ces condamnations avec tous les autres courants fondamentalistes ou réformistes. Cependant, le personnage de Mirzâ Ghulâm Ahmad, sa qualité de prophète et les réincarnations qui se manifestent en sa personne, sont fort éloignés de l'islam des débuts. Nous qualifierons les Ahmadis de "fondamentalistes non orthodoxes et modernistes".

Si la Abmadiyya fustige ce qu'elle estime être le relâchement des mœurs en Occident, elle accepte toutes les nouvelles technologies et défend la compatibilité de l'islam avec le progrès scientifique et technique, ce qui ne constitue pas une spécificité de ce courant. Elle a trouvé une figure emblématique en la personne du Docteur Abdus Sallam, prix Nobel de physique en 1979, humaniste, modeste et très pieux. Si, selon Olivier Roy: "On peut définir la modernité en termes de valeurs (celles de la démocratie). Ou bien on peut définir la modernité dans le sens du détachement de l'individu des identités et des appartenances naturelles

13. Responsable national de la mission, il est aussi l'imâm.

14. Tract «Le Messie promis et Mahdi est venu! Que signifie Ahmadiyyat».

15. Rompre avec cette pratique revient à s'exclure de son groupe d'appartenance. La participation aux cérémonies est la preuve que malgré le processus d'individualisation, on reste membre de son lignage, ou de son village (MARIE, 1994).

16. On appelle alfa les lettrés coraniques, qualité que leur dénient les "nouveaux docteurs " rentrés des universités du monde arabe. 
et de l'affirmation du sujet ${ }^{17}$, la modernité de la Ahmadiyya ne fait aucun doute, elle partage avec l'islam confrérique au Bénin l'attachement à la démocratie - alors que les autres courants fondamentalistes dénoncent l'importation du modèle occidental honni - et elle participe du mouvement général d'individualisation de la pratique religieuse. Le Ahmadi, tout en privilégiant les valeurs prônées par la religion, cultivera ses capacités et aura une vie sociale et professionnelle bien remplie; les luxueuses voitures garées sur le parking de la place 'îdî̀ à l'extérieur de Porto-Novo attestent de la réussite sociale d'une partie des membres présents.

La Abmadiyya prône une religion universelle dans laquelle elle rassemble les données des grandes religions et milite pour le rapprochement avec celles-ci, y compris avec le vaudou. Quand le pape a appelé au dialogue entre les religions, les Ahmadis ont rencontré les prêtres et les pasteurs avec lesquels ils entretiennent de meilleurs rapports qu'avec les imâms. Il semblerait que ce soient plutôt les autres musulmans, chez lesquels ils fustigent « les mollahs ignorants » qui refusent le dialogue avec eux en application de leur éviction de l'Umma en 1974. Pour mener à bien ses actions, le groupe béninois de la Abmadiyya, inséré dans un réseau transnational à la structure centralisée et très hiérarchisée, se trouve soumis à l'autorité du chef temporel et spirituel, et bénéficie des moyens dont dispose ce réseau puissant.

\section{Une organisation pyramidale très hiérarchisée}

À sa tête, un calife : le $5^{\mathrm{e}}$ calife, Hazrat Mirza Mazroor Ahmad, a remplacé en 2003 Hazrat Mirza Tahir Ahmad, comme "Chef suprême de la Jamâ'at islamique Abmadiyya ". La Abmadiyya qui, en 1947 avait opté pour le Pakistan, y fait l'objet de persécutions de la part des "orthodoxes" depuis les années 1950 ; exclue de l'Umma en 1974, elle y est réprimée officiellement depuis 1984, date à laquelle le calife s'est exilé à Londres d'où il exerce une autorité absolue et centralise les cotisations des fidèles du monde entier. C'est lui qui donne les directives et, une fois par an, réunit en Jalsa Salana des délégations de chaque pays.

L'amîr, responsable de la Abmadiyya dans un pays ou pour un groupe de pays, coordonne les actions à l'échelle nationale et entretient les relations publiques avec les instances politiques. Il est à la fois le responsable religieux, l'ambassadeur du calife et le directeur du site local d'une organisation transnationale. L'amîr Assan Secundar, Mauricien d'origine pakistanaise, en poste à Porto-Novo lors de ma première mission de recherche, a été remplacé en juillet 2004 par l'ancien missionnaire de Parakou, Khalid Maamoud, arrivé du Pakistan en septembre 2001. Des missionnaires sont affectés dans les villes secondaires à partir desquelles ils couvrent une zone. Responsables de l'organisation dans leur

17. Roy, 2004. 
secteur, ils travaillent sous le contrôle de l'amir. Selon ce dernier, il y aurait dix missions principales au Bénin, dont dépendraient trente-cinq missions de niveau inférieur ${ }^{18}$.

Dans chaque localité où la Abmadiyya installe une mission, les membres sont organisés en groupes non mixtes par tranche d'âge : sept-quinze ans, quinzequarante ans, plus de quarante ans pour les garçons, et pour les filles sept-quinze ans et plus de quinze ans; chaque groupe se trouve placé sous l'autorité d'un responsable. Les jeunes doivent étudier le Coran, connaître les autres religions et effectuer un travail humanitaire ; à partir de l'âge de quinze ans, ils encadrent des colonies de vacances et enseignent le Coran aux enfants. Il ne suffit pas d'apprendre le Coran, il faut s'investir dans des activités permettant le contact avec les autres. Chaque adepte est encadré et encadre à son tour le groupe de niveau inférieur. Il n'y a pas de possibilité de penser hors du champ de la Ahmadiyya, et l'obéissance aux responsables est un devoir absolu. Dans l'éditorial du bulletin Le Guide des derniers Temps de décembre 2004, le responsable de la publication appelle ses lecteurs à faire leur bilan spirituel: «Est-ce que vous avez été juste envers votre Dieu ? Est-ce que vous n'avez pas mal agi concernant votre travail ? Est-ce que vous avez obéi aux responsables de la communauté ? Est-ce que vous étiez en parfaite entente avec les différents responsables de la Jamâ'at islamique Amadiyya?"

\section{Les actions de communication et de prosélytisme}

Les missionnaires et ceux qui exercent des responsabilités reçoivent une rémunération grâce à l'argent des cotisations.

La Abmadiyya dispose de moyens financiers importants : chaque adepte verse un seizième de son salaire ou de ses revenus; les missions d'Afrique reçoivent de l'argent de Londres, les Ahmadis des pays développés financent partiellement l'action missionnaire et humanitaire menée dans les pays du Sud ${ }^{19}$. La Abmadiyya agit dans deux domaines : la da'wa, et au service de celle-ci, l'action humanitaire. La da'wa se réalise dans le travail missionnaire, le prosélytisme, mais également dans la construction de mosquées. La Abmadiyya s'est donné comme projet, sous forme de slogan, "cent mosquées pour le Bénin ». Le travail humanitaire constitue le second volet de l'action et à cette fin, le calife a fondé, en 1995, l'ONG « Humanity first », enregistrée au Bénin en 2003.

Pour la da'wa, les Ahmadis utilisent les moyens de communication les plus modernes : sites internet, transmission par satellite depuis Londres des sermons du calife captés au Bénin par les antennes paraboliques, prêches traduits dans

18. Entretien avec l'amîr Khaled Maamoud, 27 janvier 2005.

19. La Ahmadiyya a fait de nombreux adeptes aux États-Unis (MOHAMMAD-ARIF, 2000, p. 45-48), elle est également présente en Europe. Les adeptes de ces pays développés versent leur contribution, et tous les versements sont centralisés par le calife. 
les langues locales, sonorisation des sermons, interventions dans la presse et dans les radios locales. Ils disposent de leurs propres moyens de publication : ils diffusent au Bénin un journal mensuel en français, le Message, imprimé à l'Île Maurice, qui édite les sermons du calife, et un bulletin imprimé localement : Le Guide des Derniers Temps, ci-dessus mentionné. Entrepreneur religieux dynamique, comme tout responsable d'ONG ou chef d'entreprise, de son bureau relié au monde, l'amîr gère avec autorité sa mission et son personnel. Il s'est tissé un réseau de relations locales bien choisies tout en restant connecté en permanence avec le réseau mondial de l'organisation. La Abmadiyya a la capacité de mener des campagnes de communication. Prenons l'exemple de sa propre promotion par le biais de la figure emblématique du docteur Abdus Salam : en 2002, la photographie de ce dernier illustrait le calendrier de la Ahmadiyya imprimé au Bénin, les postes béninoises ont sorti un timbre à son effigie, et l'on peut penser que la Ahmadiyya n'y est pas étrangère. L'amîr Essan Secundar a tenu une conférence de presse pour le présenter et le journal Le Béninois en a rendu compte sur une page entière, la vie exemplaire du Professeur est rappelée sur les sites internet.

Pour ses expéditions de prosélytisme, la Abmadiyya s'est dotée d'un bon équipement : les groupes, non mixtes, partent dans les villages en véhicules " tous terrains " avec sonorisation, y restent plusieurs jours, allient prédication et aide à la population. Chaque groupe s'adresse à une population de la même classe d'âge et du même sexe. En ville, les femmes s'installent sous un appatam ${ }^{20}$ pour établir le contact avec les passantes. Dès le début, la Ahmadiyya s'était inspirée des méthodes des missionnaires protestants que Mirzâ Ghulâm Ahmad avait vus à l'œuvre: envoi de missionnaires dans le monde entier, distribution de tracts et de brochures, publication de revues. Dans le contexte des villes côtières où le religieux atteint une grande visibilité, les expéditions et le prosélytisme en pleine rue rappellent les croisades des mouvements pentecôtistes et évangéliques. Une similitude est remarquable également dans l'occupation d'espaces profanes à des fins religieuses : de même que des groupes évangélistes et pentecôtistes louent des salles ou des stades pour des rassemblements, les missionnaires de la Ahmadiyya organisent des tournois de football afin d'entrer en contact avec les jeunes garçons. Le porte-à-porte également pratiqué ne se révèle pas toujours efficace : à Porto-Novo, les femmes l'ont arrêté parce que la population les accueillait mal et les confondait avec les Témoins de Jéhovah.

Dans le domaine humanitaire, comme d'autres ONG islamiques ${ }^{21}$, Humanity First porte son effort sur la santé : elle a construit une importante clinique à

20. Abri au toit de palme tenu par quatre piquets, qui permet de se protéger du soleil.

21. L'Association Mondiale de l'Appel à l'Islam (ONG libyenne) a construit et assure le fonctionnement d'un hôpital à Porto-Novo et d'un centre de santé à Cotonou, le Centre Fayçal qui a été financé par le roi d'Arabie Saoudite, l'Agence des Musulmans d'Afrique (ONG koweitienne) a construit deux centres de santé dans des complexes islamiques, à Parakou et à Bassila. Il faut noter que pour un centre de santé, ces ONG construisent une cinquantaine de mosquées, dans des villages où il n’y a souvent ni école ni centre de soins. 
Porto-Novo dirigée par un médecin pakistanais, la première pierre d'un dispensaire a été posée à Allada et Thuy, un hôpital est prévu à Parakou. En outre, elle organise, comme d'autres ONG islamiques, des caravanes de santé : une équipe allemande devait arriver en février 2005 pour assurer sept camps médicaux gratuits. Ces caravanes de santé permettent d'aller au-devant de la population, et en accueillant tout le monde, sans discrimination religieuse, elles s'avèrent un moyen de prosélytisme très efficace. L'éducation constitue une autre priorité, car il est nécessaire de lire et comprendre les textes et de pouvoir apporter des explications lors des prédications; la promotion de l'éducation féminine et la place de la femme dans la vie religieuse et sociale lui donnent un pouvoir d'attraction sur la population féminine, urbaine et instruite. Si l'on ajoute à cela une vie communautaire importante, être membre de la Ahmadiyya demande un engagement total. Pour mettre en œuvre leurs actions, les responsables développent des stratégies adaptées à la situation politique nationale et aux situations locales.

\section{L'islam minoritaire dans le Sud, majoritaire dans le Nord}

L'islam au Bénin, comme dans les autres pays du Golfe de Guinée, n'a pas la même histoire dans le Nord et dans le Sud. Dans le Nord, introduit par les commerçants caravaniers au plus tard au $\mathrm{XV}^{\mathrm{e}}$ siècle, il est resté jusqu'au début $\mathrm{du} \mathrm{XX}^{\mathrm{e}}$ siècle circonscrit aux quartiers des marchés. Cet islam traditionaliste ${ }^{22}$ se vit dans les confréries, la Tidjaniyya nyassiste étant aujourd'hui la plus représentée. Les conflits qui surviennent lors des successions d'imâms, n'ont rien de doctrinal, ils éclatent pour des questions de pouvoir et d'intérêt. Religion en expansion, l'islam a connu, ces dernières décennies, une forte progression en milieu rural. Cet islam confrérique subit les attaques de l'islam fondamentaliste introduit par les ONG des pays du Golfe et par les boursiers qui reviennent des universités du monde arabe. Excepté à Djougou où il occupe véritablement l'espace public par ses mosquées, ses écoles, ses bibliothèques, les femmes de plus en plus nombreuses entièrement recouvertes de noir, le fondamentalisme, bien qu'en progression, garde une influence encore limitée ${ }^{23}$. Enfin, de nombreux musulmans pratiquent leur religion hors des confréries, sans être pour autant fondamentalistes ; l'islam "se modernise ${ }^{24}$ de l'intérieur par un processus d'individualisation.

Dans le Sud, l'islam est arrivé par la côte, plus tardivement que dans le Nord, à la fin du XVIII et au début du XIX ${ }^{e}$ siècle, d'abord avec les commerçants yoruba du Nigeria, puis avec les esclaves libérés rapatriés d'Amérique et avec des commerçants hausa dont le quartier Zongo ${ }^{25}$ de Cotonou atteste la présence depuis le début du Xx ${ }^{e}$ siècle. Malgré les mosquées de plus en plus nombreuses

22. Je le qualifie de traditionaliste par rapport aux nouveaux courants qui veulent en extirper les pratiques magiques et critiquent les confréries.

23. BRÉGAND, 1999.

24. Notion employée en référence à la définition donnée précédemment (note 17).

25. Quartier peuplé de Hausa et plus globalement d'étrangers. 
et de plus en plus imposantes, la visibilité de l'islam reste modeste par rapport à celle des mouvements chrétiens de tous ordres. Hormis le fondamentalisme d'inspiration saoudienne ${ }^{26}$, c'est du Nigeria et par la côte que les nouveaux courants musulmans arrivent au Bénin, installent leur siège à Porto-Novo, gagnent Cotonou, puis tentent une percée dans le Nord, d'abord à Parakou avant de rayonner dans les villages.

Ainsi à Porto-Novo trouve-t-on la Abmadiyya depuis les années 1960, avec une éviction durant le régime du PRPB, le Tabligh depuis 1986 et deux courants mystiques récents : l'ordre Nímatullâhî dont le représentant au Bénin est Yacoubou Fassassi, haut fonctionnaire de la Banque mondiale, conseiller auprès du Président de la République, et la 'Alawiyya introduite par un disciple de Cheikh Bentounès. Ces mouvements musulmans trouvent facilement leur place à Porto-Novo où le nombre des Églises chrétiennes est encore plus important. La côte, très tournée vers l'extérieur - Lagos, Lomé, Accra sont peu distantes - accueille les nouveaux mouvements religieux, comme cela se passe dans toutes les grandes villes côtières du Golfe de Guinée. Dans le Nord où l'islam est plus ancien, les nouveaux courants rencontrent plus de résistance de la part des musulmans; il arrive même qu'ils soient chassés comme ce fut, à Parakou, le cas de tablìghî en sortie de da'wa.

\section{La stratégie de la Ahmadiyya dans le contexte politique béninois}

\section{Séduction des rois et relations publiques}

Présente dans le Sud du pays dès les années 1960, la Ahmadiyya a fait construire en 1974 sa mosquée à Porto-Novo. À Cotonou, elle disposait « d'une humble mosquée au bord de la lagune ${ }^{27}$ que Kerekou a fait fermer en 1987. Aujourd'hui, cette affaire ressort. Que s'était-il passé ? Voici l'explication de la Abmadiyya : lorsque le Docteur Abdus Salam, Prix Nobel de physique, est venu au Bénin invité par Kerekou en janvier 1987, les imâms de Cotonou avaient préparé la grande mosquée pour la prière et, lorsque la voiture officielle est venue le chercher, le Docteur Abdus Salam a demandé s'il n'y avait pas une mosquée Ahmadiyya, il s'est fait conduire dans l'humble mosquée au bord de la lagune et a prié avec les Ahmadis. Les imâms vexés et indignés sont intervenus auprès de Kerekou et ont obtenu la fermeture de la mosquée.

Dans le Nord, pendant le régime de la Révolution, les musulmans de Parakou ont fait chasser le missionnaire par ordre du gouvernement, refusant à Mirzâ

26. Ces fondamentalistes (diplômés des universités arabes et membres des ONG du Golfe) se disent sunnites, refusent l'adjectif "wahhabite ", ce que d'ailleurs ils ne sont pas vraiment, ils correspondent plus justement à la mouvance qu'Olivier Roy appelle néo-fondamentaliste (Roy, 2002).

27. Récit de l'amîr en février 2002. 
Ghulâm Ahmad la qualité de prophète, très certainement en application des décisions prises à l'encontre de la Abmadiyya ${ }^{28}$.

Aujourd'hui, la liberté des cultes est assurée; la Abmadiyya a donc le droit de louer une maison pour installer sa mission, et de construire une mosquée dès lors qu'elle possède un terrain. La difficulté est alors d'obtenir le terrain, car les autres musulmans tentent d'intervenir dans les circonscriptions urbaines pour que le terrain ou le permis de construire leur soit refusé. De plus, une véritable compétition s'est engagée entre les différents courants religieux et entre les ONG islamiques pour l'achat où l'octroi de terrains. La stratégie de la Ahmadiyya a reposé sur la séduction des chefs traditionnels. En 2001, trois rois : d'Allada, de Savé et de Parakou ${ }^{29}$, sont partis à Londres à l'Assemblée annuelle (Jalsa sâlâna), tous frais payés, car en tant que rois ils devaient rencontrer le calife : le calendrier de l'année 2002 publie la photographie de la rencontre. Le calife leur a offert à chacun un morceau de tissu du vêtement que portait Mirzâ Ghulâm Ahmad, désormais encadré et exposé dans le palais de chacun d'entre eux. L'appartenance supposée des rois à la Abmadiyya est d'un grand effet médiatique, ils peuvent influencer la population, et surtout leur intercession auprès des pouvoirs publics a permis d'obtenir le terrain jusque-là refusé.

\section{Dans le Sud, la floraison des courants religieux}

À Porto-Novo, plusieurs familles ont rejoint la Abmadiyya dès les années 1960. Le jour de Tabaski 2002, comme à l'occasion de toutes les fêtes du calendrier musulman, se tenait à neuf heures le grand rassemblement pour la prière sur la place 'îdî. À l'extérieur de la ville, la place 'îdî de la Abmadiyya occupe une clairière. Les hommes et les femmes font leurs ablutions ensemble, une paroi en roseaux sépare en deux parties l'aire de prière recouverte de nattes : d'un côté les femmes et les petites filles, de l'autre les hommes et les jeunes garçons. La séance a commencé par une prière d'environ un quart d'heure dirigée par l'amîr, et s'est poursuivie par son sermon, d'une durée de trois quarts d'heure, prononcé en français, amplifié et traduit en yoruba. Au total, il y avait entre soixante-dix et quatre-vingts personnes et même si tous les adeptes n'étaient pas présents, on peut supposer que la majorité des membres s'étaient déplacés à l'occasion de la fête de Tabaski.

Les femmes présentes à cette occasion, très engagées dans les œuvres de la Abmadiyya, exercent une activité professionnelle et sont instruites. L'une d'elle, institutrice chargée de la formation des maîtres, s'occupe à la Ahmadiyya d'un

28. Entretien du 26 février 2002 avec le Président de l’Union islamique du Borgou. Fondée en 1984, l'Union Islamique est l'interface entre les pouvoirs publics et les musulmans.

29. Ce dernier est décédé en octobre 2004. La Abmadiyya lui rend hommage dans son bulletin Le Guide de décembre 2004. On y apprend qu'il avait rejoint la Abmadiyya dès 2000 et avait tous les ans, jusqu'en 2003, été invité à la Jalsa sâlâna à Londres et en Allemagne. 
centre de formation pour jeunes filles où, faute de moyens suffisants, on leur apprend la couture et la restauration, d'autres sont commerçantes, infirmières. Tout ceci confirme le souci d'éducation et la place des femmes à la Abmadiyya. Contrairement aux fondamentalistes de la mouvance wahhabite qui prônent leur réclusion, la Abmadiyya encourage leur promotion sociale, mais elles évoluent dans un cadre strict : ainsi une jeune femme yoruba, commerçante à Cotonou, a refusé l'invitation à la 'aqîqa de l'enfant de sa sœur qui n'est pas mariée, " car chez les musulmans yoruba, cela ne pose aucun problème qu'une femme musulmane ait des enfants sans être mariée ", explique-t-elle; c'est au nom de la morale qu'elle a pris des distances, mais elle a envoyé de l'argent à sa sœur par souci humanitaire. Elle insiste beaucoup sur la nécessité de se voiler pour sortir, mais déplore que les autres musulmanes se moquent des femmes voilées. Pour sortir, les femmes de la Abmadiyya se couvrent la tête d'un léger voile, ne cachant pas leur visage, ce détail différencie la branche de Qadian de la branche de Lahore ${ }^{30}$. Les Ahmadis, comme tous les fondamentalistes, veulent extraire l'islam de son ancrage culturel; dans le Sud du Bénin, l'islam se vit en harmonie avec les cultures yoruba ou apparentées. En outre, le dernier témoignage montre que, bien qu'ils se montrent ouverts au dialogue, leur rigorisme peut les conduire à des attitudes sectaires par rapport aux autres musulmans.

Ces femmes trouvent leur place dans la vie religieuse et sociale du groupe. Mais ceci n'est pas spécifique à la Ahmadiyya, car dans les villes côtières, les femmes jouent un rôle économique important, essentiellement dans le commerce ; les Alhadja ${ }^{31}$, qui font construire des immeubles et des mosquées à l'instar des transporteurs musulmans du Nord, affichent par là leur statut et se constituent un capital social. Ce que la Ahmadiyya apporte en plus aux femmes, c'est la valeur accordée à l'éducation, la possibilité d'étudier les textes religieux, et de développer l'échange intellectuel ${ }^{32}$. Plusieurs femmes, chrétiennes à l'origine et converties par leur mari, ont fait état des conversations que permettait au sein de leur couple la comparaison entre la Bible et le Coran. À Cotonou, où elle a fait construire une mosquée de grandes dimensions près du marché, la Abmadiyya recrute chez les chrétiens, et particulièrement chez les Témoins de Jéhovah. Ce type de conversions s'insère dans un phénomène plus général de nomadisme

30. Catherine Servan-Schreiber (1999) remarque qu'au Surinam où les Ahmadis de la branche de Lahore représentent un tiers des musulmans, les femmes ne portent pas le voile, alors qu'à l'île Maurice où la Ahmadiyya se rattache à la branche de Qadian, de plus en plus de femmes se voilent (C. SERVAn-SchreIBER, 1998).

31. On désigne par ce nom de catégorie, les femmes qui ont fait le pèlerinage et ont une visibilité sociale importante, à l'instar des Alhadji du Nord (BRÉGAND, 1998) Un tel statut de la femme musulmane serait impensable dans le Nord. En 2005, j'ai visité le complexe islamique qu'al-Hadja Naïma Toukourou, commerçante au marché Dantokpa, est en train de faire construire, et qui, outre une magnifique mosquée presque terminée, comprendra une école, un orphelinat, un centre de formation.

32. Même observation chez les femmes de la Abmadiyya au Surinam (SERvan-SchreIBER, 1999). 
religieux. L'ancien président de la République, Mathieu Kerekou, d'abord entouré de devins du vaudou et de "marabouts ", puis converti au pentecôtisme, en offre un exemple, il n'est pas rare de passer d'un groupe à l'autre, particulièrement dans les nouveaux groupes protestants. Les Ahmadis ont de meilleurs rapports avec les chrétiens qu'avec les musulmans, ils vont à des enterrements chrétiens et invitent ceux-ci à entrer dans leurs mosquées. "L'important est d'entrer en contact avec eux ", cette déclaration revient de façon récurrente au cours des entretiens. Les chrétiens qui se convertissent ne vivent pas une rupture complète, puisqu'ils continuent de lire la Bible, certains gardent même leur prénom.

Les conversions entraînent parfois des attitudes de rejet de la part des familles, mais les problèmes s'aggravent lorsqu'il s'agit d'une famille musulmane. Dans tous les cas, le nouvel adepte trouve une communauté d'accueil, et s'il connaît des difficultés, le groupe lui viendra en aide, car «la communauté apporte un soutien moral et matériel à ses membres qui traversent des périodes difficiles ${ }^{33}$. La vie communautaire est importante : une réunion mensuelle, une fête annuelle, des actions à l'intérieur de sa classe d'âge, des sorties en groupe, une prise en charge collective quand un membre a des problèmes, des réunions à diverses occasions. Cette vie communautaire, comme les méthodes de prédication, rappelle les groupes évangéliques et pentecôtistes. Les motivations de ceux qui adhèrent au mouvement sont certainement semblables. Cette nouvelle appartenance participe au processus d'individualisation dans la mesure où elle permet de sortir de la logique des différentes communautés d'origine (logique de la dette, des cérémonies ruineuses, des sollicitations sans fin en direction de celui qui réussit socialement ${ }^{34}$ ), tout en proposant le soutien d'une nouvelle communauté. La Abmadiyya dans le Sud recrute beaucoup dans les milieux aisés ; ces derniers y trouvent de nouvelles normes de solidarité grâce auxquelles ils échappent à l'obligation de redistribution sans fin.

Revenons à la conversion du roi d'Allada. Ce roi diplômé d'économie a accepté le trône après 1991. Son chargé de mission à la communication affirme que Sa Majesté, bien que fréquentant la Abmadiyya, joue toujours un rôle dans les cultes vaudous. Il a donné un terrain pour la construction de la mosquée, a participé le 16 février 2002 à l'inauguration qui a rassemblé des catholiques, les chrétiens célestes, les protestants et les adeptes du vaudou. Durant la cérémonie, l'amîr de Porto-Novo a appelé les musulmans à aider les catholiques, le journal Le Béninois a consacré une page à l'événement. La Abmadiyya qui prône une religion universelle dont Mirzâ Ghulâm Ahmad serait "le Messie promis et Mahdi ", mise plus sur le dialogue interreligieux que sur une possibilité de rapprochement avec les autres musulmans. Il faut ajouter que dans le Sud, il y a des interférences entre les religions : grand prêtre du vaudou et cependant

33. Madame Daouda A., sage-femme à la retraite depuis dix ans, responsable des femmes à Porto-Novo.

34. MARIE, 1997. 
détenteur d'un morceau du vêtement de Mirzâ Ghulâm Ahmad, le roi d'Allada négocie avec les uns et les autres, il est d'abord un homme d'affaires.

Dans les villes du Sud, la Abmadiyya a trouvé « un espace» pour exercer ses activités à la faveur des libertés publiques. Elle n'est certes pas reconnue par les autres musulmans, mais l'exclusion qui la frappe est moins violente que dans le Nord. De plus, le foisonnement d'églises et de mouvements religieux contribue à sa banalisation. Un groupe de Ahmadis prêchant sous un appatam participe comme les autres à l'occupation physique et sonore de l'espace public par le religieux.

\section{Dans le Nord, travail humanitaire et prosélytisme}

La Abmadiyya avait été chassée de Parakou pendant le régime de la Révolution. Un missionnaire, originaire de Karimâma (extrême Nord du Bénin) et formé au Nigeria s'installe en octobre 1997 dans une modeste case ; en septembre 2001, il est muté plus au nord et remplacé par Khalid Maamoud, dont la maison moderne et confortable atteste du rang dans la hiérarchie. Les Ahmadis revendiquent cent cinquante adeptes à Parakou, mais il n'a pas été possible d'en rencontrer un seul. Il semblerait que dans le Nord l'organisation existe, mais que le recrutement reste difficile et rare. La circonscription urbaine de Parakou a donné un terrain à la Ahmadiyya pour qu'elle construise un centre de soins et une mosquée. Les musulmans ont alors manifesté, mais le chef de la circonscription urbaine a refusé, au nom de la laïcité, de recevoir leur délégation ${ }^{35}$. Le ministre de la santé a téléphoné au président de l'Union islamique pour défendre le projet social de la Abmadiyya et lui demander de cesser de s'opposer à ce que la circonscription leur donne un terrain.

Les imâms et le président de l'Union islamique du Borgou sont catégoriques : les Ahmadis ne sont pas des musulmans, ils ne font pas partie de l'Umma. Chassés de Parakou, ils sont revenus grâce à la démocratie et à la liberté de culte et d'association, et, pour obtenir un terrain, ont corrompu le roi qui est intervenu à la circonscription urbaine : "Le roi est catholique, animiste, musulman, tout ce que l'on veut, et maintenant Abmadiyya». Cet ancien douanier devenu roi avait perdu, à la fin de sa vie, toute crédibilité aux yeux des musulmans. Selon certains responsables musulmans, la Abmadiyya aurait peut-être des appuis au gouvernement. La majorité des ministres, catholiques, n'entretiennent aucune animosité contre ce courant religieux qui appelle ses membres à se soumettre aux pouvoirs en place ${ }^{36}$. L'amîr effectue un important travail de communication

35. Information donnée par le Chef de la Circonscription urbaine en poste lorsque ces événements se sont produits. Lui-même est originaire de Parakou, catholique.

36. "Le Message" de novembre 2004 recommande : "Chaque citoyen Ahmadi est libre d'avoir son allégeance politique, mais après la joute électorale, il doit soutenir l'État et le gouvernement en place". 
en direction des instances de pouvoir. Lorsque le cinquième calife Hazrat Mirza Masroor Ahmad s'est rendu au Bénin du 4 au 10 avril 2004, il a rencontré le chef de l'État, le Président de l'Assemblée Nationale et le ministre chargé de la Famille. Il a, en outre, offert à l'Hôtel Sheraton de Cotonou, une réception à laquelle fut conviée la presse qui a très largement rendu compte de l'événement.

Les missionnaires, quant à eux, considèrent que les Ahmadis sont mieux accueillis en milieu rural, où les gens demandent des mosquées. La structure quadrille peu à peu le Nord et concentre son action sur les villages. Les responsables religieux, qui reconnaissent la percée de la Abmadiyya en milieu rural, se sont mobilisés, ils envoient leurs grands élèves coraniques faire du travail de prévention et parler de religion dans les villages. Confrontés aux nouveaux courants qui pratiquent la da'wa, qu'il s'agisse de la Abmadiyya, du Tablîgh, ou des courants d'inspiration wahhabite, les traditionalistes se sont trouvés stimulés et se sont mis eux aussi à aller prêcher pour défendre leur conception et leur pratique de l'islam.

Le journal Le Béninois du 21 février 2002 relatait l'inauguration de la mosquée de Sérékali, village assez proche de Nikki. Or, en 1997, les musulmans de Nikki avaient chassé le missionnaire. Les Ahmadis ont pu obtenir un terrain à quelque distance et inaugurer leur mosquée en collaboration avec les catholiques, mais ils continuent de se heurter à l'hostilité des musulmans. Dans un autre village du département, Sonon, la Abmadiyya voulait construire sa mosquée sur un terrain obtenu grâce à l'appui du chef, accusé lui aussi par les imâms de s'être laissé corrompre. Les musulmans se sont divisés, l'Union islamique a dû intervenir et devant l'insistance des villageois qui voulaient une mosquée, a demandé à l'Agence des Musulmans d'Afrique de la construire, ce qui fut fait. Or l'Agence des Musulmans d'Afrique, ONG koweitienne, finance des mosquées et y emploie des prédicateurs fondamentalistes, ce qui, les années précédentes, avait suscité de nombreux conflits avec les musulmans traditionalistes pour lesquels la situation devient de plus en plus difficile à contrôler. Sollicitée pour répondre aux énormes besoins de tous ordres, la Ahmadiyya, dotée d'importants moyens financiers, ne rencontre en face d'elle que les pétrodollars de l'Arabie Saoudite et du Koweit.

Comment expliquer ce relatif succès dans les villages du Nord ? Les raisons principales semblent être qu'en milieu rural, les conversions sont récentes et ces nouveaux musulmans, qui vivent dans un environnement culturel marqué par les religions traditionnelles peuvent accepter Mirzâ Ghulâm Ahmad sans réticence. Vivant pour la plupart dans une grande pauvreté, ils perçoivent la Abmadiyya comme une ONG dont ils espèrent une aide. En milieu rural, son action repose sur le travail humanitaire, ainsi l'inauguration d'une mosquée est-elle toujours accompagnée de dons utiles à la population : à Sérékali, à l'occasion de l'inauguration de la mosquée, la Ahmadiyya a livré des tricycles pour handicapés. Ce sont les musulmans des villes appartenant à des familles islamisées depuis des 
générations, membres des confréries, qui lui opposent une ferme résistance, de même que les imâms et les alfas qui, outre leur désaccord doctrinal, voient leur pouvoir et leurs sources de revenus menacés.

\section{Conclusion}

La Abmadiyya adapte son action aux situations particulières, il est certain qu'il est plus facile d'être Ahmadi en contexte d'islam minoritaire qu'en contexte d'islam majoritaire. Elle est plus implantée dans le Sud, où elle atteint quarante ans de présence et participe au mouvement général de prolifération des groupes religieux. Elle recrute essentiellement en milieu yoruba, parmi une élite sociale et intellectuelle. Les Ahmadis sont des personnes souvent instruites, capables de discuter les textes, sans doute attirées par le niveau d'éducation que promeut la Ahmadiyya, ce qui est particulièrement vrai pour les femmes. Dans le Nord où elle rencontre une forte opposition dans les villes, elle concentre son action sur le milieu rural, ce qui la conduit à changer sa pratique : elle s'adresse à des villageois illettrés pour la plupart et met l'accent sur l'action humanitaire, mais elle peut également représenter une ouverture pour les jeunes villageois. L'ironie de l'histoire, c'est que pour barrer la route à la Abmadiyya, l'Union islamique qui n'a pas les moyens de financer les mosquées dans les villages, appelle au secours l'Agence des Musulmans d'Afrique dont les imâms prêchent pour un islam fondamentaliste dont le pacifisme n'est certainement pas la principale caractéristique. Ce courant d'inspiration saoudienne qui, jusqu'à présent, avait connu des conflits avec les musulmans du Nord, profite de l'exclusion de la Ahmadiyya. Ambassadeur du calife au Bénin, entrepreneur religieux, homme de communication, totalement investi dans son action humanitaire, l'amîr a su adapter son action aux évolutions politiques en cours ; il a parfaitement compris la « politique du ventre » et en a joué. Tout en entretenant les relations publiques avec les instances politiques nationales, acteur d'un réseau transnational efficace, il a eu recours aux chefs traditionnels, symboles de la localité.

Denise BRÉGAND

Centre d'Études d'Afrique Noire - IEP de Bordeaux 
88 - Archives de sciences sociales des Religions

\section{Bibliographie}

Ajayi J. F. Ades, Crowder M., Atlas historique de l'Afrique, Paris, Éditions du Jaguar, 1988.

BANEGAS Richard, La démocratie à pas de caméléon, Paris, Karthala, 2003.

BRÉGAND Denise, Commerce caravanier et relations sociales au Bénin, Paris, L'Harmattan, 1998.

BRÉGAND Denise, «Les Wangara du Nord-Bénin face à l'avancée du fondamentalisme : Étude comparative à Parakou et Djougou ", Islam et Sociétés au Sud du Sahara, n ${ }^{\circ} 13$, 1999, p. 91-102.

FISHER Humphrey, Ahmadiyya: A Study of Contemporary Islam on the West African Coast, Londres, 1963.

FouRCHARD Laurent, MARY André, OTAYEK René, éds., Entreprises religieuses transnationales en Afrique de l'Ouest, Paris, Karthala, 2005.

Friedmann Yohanan, Prophecy Continuous; Aspects of Ahmadi Religious Thought and Its Medieval Background, Berkeley, University of California Press 1989 (2 éd. augmentée, New Delhi, Oxford University Press, 2003).

GABORIEAU Marc, "Une nouvelle prophétie musulmane : les Abmadiyya ", chapitre XXIV : "Réformes socio-religieuses et nationalisme (1870-1948)», in Claude MArkovits, dir., Histoire de l'Inde moderne, 1480-1950, Paris, Fayard, 1994, p. 551-552.

GaboriEAu Marc, «De la guerre sainte (jihâd) au prosélytisme (da'wa). Les organisations musulmanes transnationales d'origine indienne ", in Jean-Pierre BASTIAN, Françoise Champion, Kathy Rousselet, éds., La globalisation du religieux: diversité des questionnements et des enjeux, Paris, L'Harmattan, 2001, p. 35-48.

Lavan Spencer, The Ahmadiyah Movement: History and Perspective, Delhi, Manohar Book Service, 1974.

LAVAN Spencer, "Sources for Ahmadiyya history ", in W. Eric GuSTAFSON et Kenneth W. Jones, eds., Sources on Punjab history, New-Delhi, Manohar Book Service, 1975.

MARIE Alain, éd., L'Afrique des individus, Paris, Karthala, 1997.

Markovits Claude, dir., Histoire de l'Inde moderne 1480-1950, Paris, Fayard, 1994.

MAYraRgue Cédric, "Le caméléon est remonté en haut de l'arbre : le retour au pouvoir de Mathieu Kerekou au Bénin ", Politique africaine, n 62, 1996, p. 124-134.

Mayrargue Cédric, "Démocratisation politique et revitalisation politique ", in François CONSTANTIN et Christian Coulon, dirs., Religion et transition démocratique en Afrique, Paris, Karthala, 1997, p. 135-162.

Mohammad-ArIF Aminah, Salam America. L'islam indien en diaspora, Paris, CNRS Éditions, 2000.

Roy Olivier, L'islam mondialisé, Paris, Le Seuil, 2002.

Roy Olivier, "Modernisation, réformisme et réislamisation ", Mouvements, n 36, 2004, p. 22-31.

Roy Olivier, La laïcité face à l'islam, Paris, Stock, 2005.

Servan-SchreIber Catherine, «Le mouvement Abmadiyya à l'Ile Maurice» in La transmission du savoir dans le monde musulman périphérique, Lettre d'information $\mathrm{n}^{\circ} 18 \mathrm{du}$ Groupe de recherche 0122 du CNRS-EHESS, 1998, p. 40-49.

SERVAN-SCHREIBER Catherine, "Le mouvement Abmadiyya au Surinam », La transmission $d u$ savoir dans le monde musulman périphérique, Lettre d'information $\mathrm{n}^{\circ} 19 \mathrm{du}$ Groupe de recherches 0122 du CNRS-EHESS, 1999, p. 162-178. 
SERVAN-SCHREIBER Catherine, "Le Mouvement Abmadiyya : une organisation musulmane transnationale et missionnaire originale », Après-Demain (L'islam dans le monde), $\mathrm{n}^{\circ}$ 447-449, 2002, p. 8-12.

Sмiтh W. Cantwell, "Ahmadiyya ", Encyclopédie de l'Islam, $2^{\mathrm{e}}$ éd., vol. I, Leyde, E. J. Brill, 1956.

Surgy Albert de, Le phénomène pentecôtiste en Afrique Noire: le cas béninois, Paris, L'Harmattan, 2001.

Sources émanant de la Abmadiyya

Sources orales: entretiens en février 2002 et janvier 2005

Brochures

Ahmad, le Messie Promis et Mahdi, Al Shirkah Al-Islamiyya, The London Mosque, Londres, 1986.

L'accomplissement d'une grande prophétie, Islam International Publications, United Kingdom-île Maurice, 1994.

Ma Foi, par Sir Muhammad Zafrulla Khan, Ancien Président de la Cour Internationale de Justice de 1970 à 1973, Londres, s.d., 8 pages.

L'Abmadiyyat et la Philosophie du Renouveau de la Religion, par Hazrat Mirza Tahir Ahmad, Khalifatul Masih IV, The London Mosque, Londres, s.d.

Jésus en Inde, 1908.

Le bon sens à propos de la Abmadiyyat, The London Mosque, Londres, s.d.

Tracts et Presse : le Message, imprimé à l'Ile Maurice, Le Guide des Derniers Temps, bulletin local.

Sites internet: www.alislam.org; www.Ahmadiyya.info

\section{Résumé}

L'article compare les stratégies d'implantation de la Abmadiyya au Bénin, en contextes d'islam minoritaire et d'islam majoritaire. La première partie présente la Abmadiyya, mouvement fondé par Mirza Ghulam Ahmad dans l'Inde sous domination britannique. Dans un environnement religieux marqué par la progression des missions protestantes et la poussée du réformisme hindou sous l'impulsion du mouvement Arya Samaj, il se proclama mujaddid (réformateur), muhaddath (à qui Dieu parle) et mahdi (guide). Selon une étude récente, sa doctrine repose sur l'idée de la prophétie ininterrompue inspirée de la littérature soufie médiévale, particulièrement de l'œuvre d'Ibn Arabi. La seconde partie expose les vicissitudes de la Abmadiyya en fonction de l'évolution politique du pays : durant la dernière décennie, les libertés de religion et d'association lui ont permis, comme à de nombreux autres mouvements religieux, d'ouvrir des missions et de développer son prosélytisme selon des méthodes souvent comparables à celles des évangélistes. La troisième partie analyse les stratégies de la Abmadiyya, à la fois mouvement missionnaire et ONG humanitaire, qui a compris les orientations nationales et a su s'adapter aux contextes particuliers du Sud où l'islam est minoritaire et $d u$ Nord où il est majoritaire.

Mots-clés : Islam, transnationalité, prosélytisme, fondamentalisme, Mahdi, Bénin, Renouveau démocratique. 


\section{Abstracts}

The article compares the strategies used by Ahmadiyya to establish itself in Benin, both in contexts in which Islam is in the minority and in the majority. The first part presents Ahmadiyya, a movement which was founded by Mirza Ghulam Ahmad in British-dominated India. In a religious environment marked by the spread of protestant missions and by the Hindu reformist movement Arya Samaj, he proclaimed himself a mujaddid (reformer), a muhaddath (a person to whom god speaks) and mahdi (leader). According to a recent study, his doctrine is based on the idea of continuous prophecy and draws it's inspiration from medieval sufi literature, and especially of Ibn Arabi's works. The second part explains the ups and downs of Abmadiyya in relation to the political evolution of the country: during the last decade, freedom of workship and association allowed Ahmadiyya, as many other religious movements, to set up missions and to proselytise by methods which can be compared with the evangelical ones. The third part analyses Abmadiyya's strategies: it understood the national political tendencies and was able to adjust to particular contexts in the South were Islam is in the minority and in the North where it is in the majority.

Key words: Islam, transnationalism, proselytism, fundamentalism, Mahdi, Benin, Democratic Renewal.

\section{Resumen}

El articulo compara las estrategias de establecimiento de la Abmadiyya en el Benin, en dos contextos de islam minoritario y de islam mayoritario. La primera parte presenta la Abmadiyya, movimiento fundado por Mirza Ghulam Abmad en India sobre dominacion britanica. En un medio ambiente religioso, senalado por la progresion de las misiones protestantes y de la empuje del reformismo indì sobre el impulso del movimiento Arya Samaj, el se proclamo mujaddid (reformador), muhaddath (a quien Dios habla) y mahdi (guia). Segun un estudio reciente, su doctrina se apoya sobre la idea de la profecia ininterrumpida, inspirada de la literatura sufi medieval especialmente de la obra de Ibn Arabi. La segunda parte expone las vicisitudes de la Abmadiyya en funcion de la evolucion politica del pais : durante el ultimo decenio, las libertades de religion y de asociacion le han permitido, como a numerosos otros movimientos religiosos, de abrir misiones y de desarollar su proselitismo segun metodos a menudo comparable a esos de los evangelistas. La tercera parte analiza las estrategias de la Abmadiyya, a la vez movimiento misionero y ONG humanitaria, que ha entendido las orientaciones nacionales y ha sabido adaptarsea los contextos particulares de islam minoritario o mayoritario.

Palabras claves: Islam, transnacionalidad, proselitismo, fondamentalismo, Mahdi, Benin, Renacimiento democratico. 